\title{
THE FORMAL LAPLACE-BOREL TRANSFORM OF FLIESS OPERATORS AND THE COMPOSITION PRODUCT
}

\author{
YAQIN LI AND W. STEVEN GRAY
}

Received 28 July 2005; Revised 14 April 2006; Accepted 25 April 2006

The formal Laplace-Borel transform of an analytic integral operator, known as a Fliess operator, is defined and developed. Then, in conjunction with the composition product over formal power series, the formal Laplace-Borel transform is shown to provide an isomorphism between the semigroup of all Fliess operators under operator composition and the semigroup of all locally convergent formal power series under the composition product. Finally, the formal Laplace-Borel transform is applied in a systems theory setting to explicitly derive the relationship between the formal Laplace transform of the input and output functions of a Fliess operator. This gives a compact interpretation of the operational calculus of Fliess for computing the output response of an analytic nonlinear system.

Copyright @ 2006 Hindawi Publishing Corporation. All rights reserved.

\section{Introduction}

Let $u: \mathbb{R} \rightarrow \mathbb{R}$ be a function which is real analytic at a point $t_{0} \in \mathbb{R}$. Its Taylor series expansion is denoted by

$$
u(t)=\sum_{n=0}^{\infty} c_{u}(n) \frac{\left(t-t_{0}\right)^{n}}{n !} .
$$

Within its radius of convergence, $u$ is completely characterized by the sequence of coefficients $\left\{c_{u}(n)\right\}_{n=0}^{\infty}$. From this, one can construct a formal power series representation of $u$ by introducing an abstract symbol set $X_{0}=\left\{x_{0}\right\}$, namely,

$$
c_{u}=\sum_{n=0}^{\infty} c_{u}(n) x_{0}^{n} .
$$

The formal Laplace transform in this setting is the mapping

$$
\mathscr{L}_{f}: u \longmapsto c_{u}
$$

Hindawi Publishing Corporation

International Journal of Mathematics and Mathematical Sciences

Volume 2006, Article ID 34217, Pages 1-14

DOI 10.1155/IJMMS/2006/34217 
Its inverse is the formal Borel transform. When $u$ is entire, its one-sided integral Laplace transform is often well defined. Setting $t_{0}=0$, it can be written in the form

$$
\begin{aligned}
\mathscr{L}[u](s): & =\int_{0}^{\infty} u(t) e^{-s t} d t=\sum_{n=0}^{\infty} c_{\mathcal{u}}(n) \int_{0}^{\infty} \frac{t^{n}}{n !} e^{-s t} d t \\
& =s^{-1} \sum_{n=0}^{\infty} c_{u}(n)\left(s^{-1}\right)^{n},
\end{aligned}
$$

using the Laplace transformation pair

$$
t^{n} \stackrel{\mathscr{L}}{\Longleftrightarrow} n !\left(s^{-1}\right)^{n+1}, \quad n \geq 0 .
$$

Clearly, the two transforms $\mathscr{L}_{f}$ and $\mathscr{L}$ are related via

$$
\mathscr{L}[u](s)=\left.x_{0} \mathscr{L}_{f}[u]\right|_{x_{0} \rightarrow s^{-1}} .
$$

An alternative notion of the formal Laplace-Borel transform appears in [23] as a mapping between two formal power series.

The formal Laplace-Borel transform was first used by Fliess et al. for nonlinear systems analysis in $[6-8,12,19]$. A related approach was later given by Minh in [22]. In each instance, an algebraic representation of an analytic function was used to produce a type of operational calculus for computing the output responses of a nonlinear system given various analytic input functions. In [9-11] Fliess also applied the formal Laplace-Borel transform via a tensor product to examine some classical problems regarding transfer matrix representations of linear time-varying systems and generalized state space systems. Later in [3], Delaleau and Rudolph characterized the properness of linear time-varying systems using this approach. In [18], Hautus used the formal Laplace-Borel transform to characterize the invertibility properties of so-called smooth linear systems. More recently, in [2] it was applied to obtain conditions for well-posedness of the input-output mapping for several classes of boundary control systems. The formal Laplace-Borel transform applied in the analysis of linear systems, as with the analysis of input and output functions, mostly involves formal power series over a single-letter alphabet, and thus is commutative in nature. What is absent in the nonlinear systems literature is an explicit notion of computing the formal Laplace-Borel transform of an input-output operator, an idea that is familiar and useful in linear systems theory. In this setting, noncommutative formal power series arise as a natural generalization.

The main purpose of this paper is to define the formal Laplace-Borel transform of a class of analytic input-output operators using noncommutative formal power series, and then to show how this notion can provide an alternative interpretation of the operational calculus of Fliess when combined with the composition product of two formal power series $[4,5,13-16,20]$. The specific class of operators considered is known as Fliess operators. Let $X=\left\{x_{0}, x_{1}, \ldots, x_{m}\right\}$ denote an arbitrary alphabet of noncommutative letters, and $X^{*}$ the set of all words over $X$, including the empty word $\varnothing$. For each $c$ in the set of all formal power series $\mathbb{R}^{\ell}\langle\langle X\rangle\rangle$, one can formally associate a corresponding $m$-input, $\ell$-output operator $F_{c}$ in the following manner. Let $p \geq 1$ and $a<b$ be given. 
For a measurable function $u:[a, b] \rightarrow \mathbb{R}^{m}$, define $\|u\|_{p}=\max \left\{\left\|u_{i}\right\|_{p}: 1 \leq i \leq m\right\}$, where $\left\|u_{i}\right\|_{p}$ is the usual $L_{p}$-norm for a measurable real-valued function, $u_{i}$, defined on $[a, b]$ when $p \in[1, \infty)$, and $\left\|u_{i}\right\|_{\infty}=\sup _{t \in[a, b]}\left\{\left|u_{i}(t)\right|: 1 \leq i \leq m\right\}$. For any fixed $p \in[1, \infty]$, let $L_{p}^{m}[a, b]$ denote the set of all measurable functions defined on $[a, b]$ having a finite $\|\cdot\|_{p}$ norm and $B_{p}^{m}(R)[a, b]:=\left\{u \in L_{p}^{m}[a, b]:\|u\|_{p} \leq R\right\}$. With $t_{0}, T \in \mathbb{R}$ fixed, and $T>0$, define inductively for each $\eta=x_{i} \eta^{\prime} \in X^{*}$ the mapping $E_{\eta}: L_{1}^{m}\left[t_{0}, t_{0}+T\right] \rightarrow \mathscr{C}\left[t_{0}, t_{0}+T\right]$ by

$$
E_{\eta}[u]\left(t, t_{0}\right):=\int_{t_{0}}^{t} u_{i}(\tau) E_{\eta^{\prime}}[u]\left(\tau, t_{0}\right) d \tau
$$

where $E_{\varnothing} \equiv 1$ and $u_{0} \equiv 1$. The input-output operator corresponding to $c$ is the Fliess operator

$$
F_{c}[u](t):=\sum_{\eta \in X^{*}}(c, \eta) E_{\eta}[u]\left(t, t_{0}\right)
$$

All Volterra operators with analytic kernels, for example, are Fliess operators. When there exist real numbers $K, M>0$ such that

$$
|(c, \eta)|:=\max \left\{\left|(c, \eta)_{j}\right|: 0 \leq j \leq \ell\right\} \leq K M^{|\eta|}|\eta| !
$$

the formal power series $c$ is said to be locally convergent. (Here $|\eta|$ denotes the number of symbols in $\eta \in X^{*}$.) The subset of all locally convergent series is denoted by $\mathbb{R}_{L C}^{\ell}\langle\langle X\rangle\rangle$. When $c \in \mathbb{R}_{L C}^{\ell}\langle\langle X\rangle\rangle$, it is known that $F_{c}$ constitutes a well-defined operator from $B_{p}^{m}(R)\left[t_{0}, t_{0}+T\right]$ into $B_{q}^{\ell}(S)\left[t_{0}, t_{0}+T\right]$ for sufficiently small $R, S, T>0$, where the numbers $p, q \in[1, \infty]$ are conjugate exponents, that is, $1 / p+1 / q=1$ with $(1, \infty)$ being a conjugate pair by convention [17]. Therefore, the specific operator class of interest in this paper is the set of Fliess operators $\mathscr{F}:=\left\{F_{c}: c \in \mathbb{R}_{L C}^{\ell}\langle\langle X\rangle\rangle\right\}$. When $\ell=m$, this set forms a semigroup under operator composition, as does the set $\mathbb{R}_{L C}^{m}\langle\langle X\rangle\rangle$ under the composition product $[4,20]$. It will be shown here that the formal Laplace-Borel transform provides an isomorphism between these two semigroups.

The paper is organized as follows. In Section 2, the notion of a formal Laplace-Borel transform of a Fliess operator is defined. Then its basic properties are developed and a set of examples is given. In Section 3, the composition product is introduced and its relationship to the formal Laplace-Borel transform is described. Using the concepts developed in Section 3, the formal Laplace-Borel transform is shown to provide a compact interpretation of the operational calculus of Fliess in Section 4. Examples are given to illustrate the computation of the output response of an analytic system. Section 5 concludes the paper with a brief summary of the main results.

\section{The formal Laplace-Borel transform of a Fliess operator}

Consider a causal linear integral operator

$$
y(t)=\int_{t_{0}}^{t} h(t-\tau) u(\tau) d \tau
$$


where the kernel function, $h$, is analytic at $t=0$. The operator is completely characterized by the Laplace transform of the kernel function, namely, $H(s):=\mathscr{L}[h](s)=\sum_{k>0} c_{h}(k) s^{-k}$, when it exists. Therefore, the usual definition (1.3) of the formal Laplace-Borel transform over a single symbol applies directly to this case. In the more general context of Fliess operators, a generalization is required. The following preliminaries are needed to ensure the new definition is well-posed.

Theorem 2.1 [24, Corollary 2.2.4]. Suppose $c, d \in \mathbb{R}_{L C}^{\ell}\langle\langle X\rangle\rangle$. If $F_{c}=F_{d}$ on $B_{\infty}^{m}(1)\left[t_{0}, t_{0}+\right.$ $T$ ] for some finite $T>0$, then $c=d$.

Given the nested nature of the set of spaces $L_{p}\left[t_{0}, t_{0}+T\right]$ for $p=1,2, \ldots, \infty$, the following result is immediate.

Corollary 2.2. Suppose $c, d \in \mathbb{R}_{L C}^{\ell}\langle\langle X\rangle\rangle$. If $F_{c}=F_{d}$ on $B_{p}^{m}(R)\left[t_{0}, t_{0}+T\right]$ for some $p \in$ $\{1,2, \ldots, \infty\}$ and real numbers $R, T>0$, then $c=d$.

In light of this uniqueness property, there exists a one-to-one mapping between the set of well-defined Fliess operators, $\mathscr{F}$, and the set of locally convergent formal power series, $\mathbb{R}_{L C}^{\ell}\langle\langle X\rangle\rangle$. This guarantees that the following definition is well-posed.

Definition 2.3. Let $X=\left\{x_{0}, x_{1}, \ldots, x_{m}\right\}$. The formal Laplace transform is defined as

$$
\begin{aligned}
\mathscr{L}_{f} & : \mathscr{F} \longrightarrow \mathbb{R}_{L C}^{\ell}\langle\langle X\rangle\rangle \\
& : F_{c} \longmapsto c .
\end{aligned}
$$

The corresponding inverse transform, the formal Borel transform, is

$$
\begin{aligned}
\mathscr{B}_{f} & : \mathbb{R}_{L C}^{\ell}\langle\langle X\rangle\rangle \longrightarrow \mathscr{F} \\
& : c \longmapsto F_{c} .
\end{aligned}
$$

Note that when $m=0$, this definition is consistent with that given in (1.3) in the sense that a fixed function $u$ can be represented as the constant operator $F_{c_{u}}$, that is, $u=F_{c_{u}}[v]$ for all $v \in B_{p}^{m}(R)\left[t_{0}, t_{0}+T\right]$ and $\mathscr{L}_{f}[u]=c_{u}=\mathscr{L}_{f}\left[F_{c_{u}}\right]$.

It is next shown that many of the familiar properties of the integral Laplace transform also have counterparts in the present context. To facilitate the analysis, two concepts are needed.

Definition 2.4. For any $x_{i} \in X$, the left-shift operator, $x_{i}^{-1}: \mathbb{R}^{\ell}\langle\langle X\rangle\rangle \rightarrow \mathbb{R}^{\ell}\langle\langle X\rangle\rangle$, is defined as

$$
x_{i}^{-1}(c)=\sum_{\eta \in X^{*}}(c, \eta) x_{i}^{-1}(\eta)
$$

where

$$
x_{i}^{-1}(\eta)= \begin{cases}\eta^{\prime}: \eta=x_{i} \eta^{\prime}, & \eta^{\prime} \in X^{*} \\ 0: & \text { otherwise }\end{cases}
$$


Definition 2.5. A Dirac series, $\delta_{i}$, is a generalized series with the defining property that $F_{\delta_{i}}[u]=u_{i}(t)$ for any $1 \leq i \leq m$.

The main result concerning elementary properties of the formal Laplace-Borel transform is stated below.

Theorem 2.6. Given any $c, d \in \mathbb{R}_{L C}^{\ell}\langle\langle X\rangle\rangle$ and scalars $\alpha, \beta \in \mathbb{R}$, the following identities hold.

(1) Linearity:

$$
\begin{aligned}
\mathscr{L}_{f}\left[\alpha F_{c}+\beta F_{d}\right] & =\alpha \mathscr{L}_{f}\left[F_{c}\right]+\beta \mathscr{L}_{f}\left[F_{d}\right], \\
\mathscr{B}_{f}[\alpha c+\beta d] & =\alpha \mathscr{P}_{f}[c]+\beta \mathscr{B}_{f}[d] .
\end{aligned}
$$

(2) Integration:

$$
\begin{aligned}
& \mathscr{L}_{f}\left[I^{n} F_{c}\right]=x_{0}^{n} c, \\
& \mathscr{B}_{f}\left[x_{0}^{n} c\right]=I^{n} F_{c},
\end{aligned}
$$

where $I^{n}(\cdot)$ is the $n$th order integration operator so that

$$
I^{n} F_{c}[u](t)=\int_{0}^{t} \int_{0}^{\tau_{1}} \cdots \int_{0}^{\tau_{n-1}} F_{c}[u]\left(\tau_{n}\right) d \tau_{n} \cdots d \tau_{2} d \tau_{1}
$$

(3) Differentiation:

$$
\begin{gathered}
\mathscr{L}_{f}\left[D F_{c}\right]=x_{0}^{-1}(c)+\sum_{i=1}^{m} \delta_{i} \sqcup\left(x_{i}^{-1}(c)\right), \\
\mathscr{B}_{f}\left[x_{0}^{-1}(c)+\sum_{i=1}^{m} \delta_{i} \sqcup\left(x_{i}^{-1}(c)\right)\right]=D F_{c} .
\end{gathered}
$$

If $x_{0}^{n}$ is a left factor of $c$, that is, $c=x_{0}^{n} c^{\prime}$ for some $c^{\prime} \in \mathbb{R}_{L C}^{\ell}\langle\langle X\rangle\rangle$, then

$$
\begin{aligned}
& \mathscr{L}_{f}\left[D^{n} F_{c}\right]=x_{0}^{-n}(c), \\
& \mathscr{B}_{f}\left[x_{0}^{-n}(c)\right]=D^{n} F_{c},
\end{aligned}
$$

where $D^{n}(\cdot)$ is the nth-order differentiation operator so that $D^{n} F_{c}[u](t)=d^{n} F_{c}[u](t)$ / $d t^{n}$, and $\sqcup$ denotes the shuffle product (see, e.g., [21]).

(4) Multiplication:

$$
\begin{gathered}
\mathscr{L}_{f}\left[F_{c} \cdot F_{d}\right]=\mathscr{L}_{f}\left[F_{c}\right] \sqcup \mathscr{L}_{f}\left[F_{d}\right], \\
\mathscr{B}_{f}[c \sqcup d]=\mathscr{B}_{f}[c] \cdot \mathscr{B}_{f}[d] .
\end{gathered}
$$

Proof. The properties of linearity and integration are trivial. The multiplication property follows from results in the literature concerning the shuffle product $[22,24]$. Only the differentiation property remains to be justified. It is shown in [24] that the derivative of 
6 The formal Laplace-Borel transform of Fliess operators

a Fliess operator is

$$
\frac{d}{d t} F_{c}[u](t)=F_{x_{0}^{-1}(c)}[u](t)+\sum_{i=1}^{m} u_{i}(t) F_{x_{i}^{-1}(c)}[u](t) .
$$

Applying the formal Laplace transform to this equality gives the first pair of equations in part (3). Now if $x_{0}$ is a left factor of $c$, then $F_{x_{i}^{-1}(c)}[u](t)=0$ for $i=1,2, \ldots, m$. In this case, $d F_{c}[u](t) / d t=F_{x_{0}^{-1}(c)}[u](t)$. Proceeding inductively, the second pair of equations follows.

The following definition is utilized in the examples which follow.

Definition $2.7[1]$. Let $c \in \mathbb{R}\langle\langle X\rangle\rangle$ be proper (i.e., $(c, \varnothing)=0$ ). Then the star operator applied to $c$ is defined as

$$
c^{\star}:=\sum_{n \geq 0} c^{n}:=(1-c)^{-1}
$$

where $c^{n}$ denotes the catenation power.

Observe that when $c$ is not proper, it is always possible to write $c=(c, \varnothing)\left(1-c^{\prime}\right)$. In which case, there exists a $c^{-1} \in \mathbb{R}\langle\langle X\rangle\rangle$ such that under the catenation product $c c^{-1}=1$ and $c^{-1} c=1$. Specifically,

$$
c^{-1}=\frac{1}{(c, \varnothing)}\left(1-c^{\prime}\right)^{-1}=\frac{1}{(c, \varnothing)}\left(c^{\prime}\right)^{\star} .
$$

Example 2.8. Let $X=\left\{x_{0}, x_{1}, x_{2}\right\}$ and $F_{c}[u](t)=\exp \left(\int_{0}^{t} u_{1}(t)+u_{2}(t) d t\right)$. Observe that $F_{c}[u]$ can be expanded as

$$
\begin{aligned}
F_{c}[u](t)= & \sum_{n \geq 0} \frac{1}{n !}\left(\int_{0}^{t} u_{1}(t)+u_{2}(t) d t\right)^{n} \\
= & \sum_{n \geq 0} \int_{0}^{t}\left[u_{1}\left(\tau_{1}\right)+u_{2}\left(\tau_{1}\right)\right] \int_{0}^{\tau_{1}}\left[u_{1}\left(\tau_{2}\right)+u_{2}\left(\tau_{2}\right)\right] \\
& \times \cdots \int_{0}^{\tau_{n-1}}\left[u_{1}\left(\tau_{n}\right)+u_{2}\left(\tau_{n}\right)\right] d \tau_{n} \cdots d \tau_{2} d \tau_{1} .
\end{aligned}
$$

Therefore,

$$
\mathscr{L}_{f}\left[F_{c}\right]=\sum_{n \geq 0}\left(x_{1}+x_{2}\right)^{n}=\left(x_{1}+x_{2}\right)^{\star}
$$

This result can be viewed as an operator generalization of the integral transform pair

$$
e^{t} \stackrel{\mathscr{L}}{\Longleftrightarrow}(1-s)^{-1} \text {. }
$$

Other formal Laplace-Borel transform pairs are given in Table 2.1. (See [24, Example 2.3.9] for additional discussion related to this example.) 
Table 2.1. Some formal Laplace-Borel transform pairs.

\begin{tabular}{lc}
\hline$F_{c}$ & $\mathscr{L}_{f}\left[F_{c}\right]$ \\
\hline$F_{c}: u \longmapsto 1$ & 1 \\
$F_{c}: u \longmapsto t^{n}$ & $n ! x_{0}^{n}$ \\
$F_{c}: u \longmapsto\left(\sum_{i=0}^{n-1} \frac{\left(\begin{array}{c}i \\
n-1\end{array}\right)}{i !} a^{i} t^{i}\right) e^{a t}$ & $\left(1-a x_{0}\right)^{-n}$ \\
$F_{c}: u \longmapsto \frac{1}{n !}\left(\int_{t_{0}}^{t} \sum_{j=1}^{k} u_{i_{j}}(\tau) d \tau\right)^{n}$ & $\left(x_{i_{1}}+x_{i_{2}}+\cdots+x_{i_{k}}\right)^{n}$ \\
$F_{c}: u \longmapsto \sum_{n \geq 0} \frac{a_{n}}{n !}\left(\int_{t_{0}}^{t} \sum_{j=1}^{k} u_{i_{j}}(\tau) d \tau\right)^{n}$ & $\sum_{n \geq 0} a_{n}\left(x_{i_{1}}+x_{i_{2}}+\cdots+x_{i_{k}}\right)^{n}$ \\
$F_{c}: u \longmapsto \exp \left(\int_{t_{0}}^{t} \sum_{j=1}^{k} u_{i_{j}}(\tau) d \tau\right)$ & \\
$F_{c}: u \longmapsto \int_{t_{0}}^{t} \sum_{j=1}^{k} u_{i_{j}}(\tau) d \tau \exp \left(\int_{t_{0}}^{t} \sum_{j=1}^{k} u_{i_{j}}(\tau) d \tau\right)$ & $\frac{\left(x_{i_{1}}+x_{i_{2}}+\cdots+x_{i_{k}}\right)^{\star}}{\left[1-\left(x_{i_{1}}+x_{i_{2}}+\cdots+x_{i_{k}}\right)\right]^{2}}$ \\
$F_{c}: u \longmapsto \cos \left(\int_{t_{0}}^{t} \sum_{j=1}^{k} u_{i_{j}}(\tau) d \tau\right)$ & $\frac{1}{1+\left(x_{i_{1}}+x_{i_{2}}+\cdots+x_{i_{k}}\right)^{2}}$ \\
$F_{c}: u \longmapsto \sin \left(\int_{t_{0}}^{t} \sum_{j=1}^{k} u_{i_{j}}(\tau) d \tau\right)$ & $\frac{x_{i_{1}}+x_{i_{2}}+\cdots+x_{i_{k}}}{1+\left(x_{i_{1}}+x_{i_{2}}+\cdots+x_{i_{k}}\right)^{2}}$ \\
\hline
\end{tabular}

Example 2.9. Let $X=\left\{x_{0}, x_{1}, \ldots, x_{m}\right\}$. Suppose $F_{c}$ has the generating series $c=\sum_{\eta \in X^{*}} \eta$, and $F_{\xi}$ is given for some fixed word $\xi \in X^{*}$. Then

$$
\begin{aligned}
\mathscr{L}_{f}\left[F_{c} \cdot F_{\xi}\right] & =\mathscr{L}_{f}\left[F_{c}\right] \sqcup \mathscr{L}_{f}\left[F_{\xi}\right]=c \sqcup \xi \\
& =\sum_{\nu \in X^{*}}\left(\begin{array}{l}
\nu \\
\xi
\end{array}\right) \nu
\end{aligned}
$$

where $(\stackrel{v}{\xi})$ denotes the number of subwords of $\nu$ which are equal to $\xi$ (see [21, page 127]).

\section{The formal Laplace-Borel transform and the composition product}

In this section, the formal Laplace-Borel transform of the composition of two Fliess operators is considered. This leads to an important semigroup isomorphism between the set of all Fliess operators and the set of all locally convergent formal power series with compatible dimensions. In system theory applications, this analysis is useful for models consisting of cascaded nonlinear input-output systems. The definition of the composition product first appeared in $[4,5]$. Its set of known properties was significantly expanded in $[13-17,20]$. The definition is constructed recursively in terms of the shuffle product. 
8 The formal Laplace-Borel transform of Fliess operators

Definition 3.1. For any $c \in \mathbb{R}^{\ell}\langle\langle X\rangle\rangle$ and $d \in \mathbb{R}^{m}\langle\langle X\rangle\rangle$, the composition product is defined as

$$
c \circ d=\sum_{\eta \in X^{*}}(c, \eta) \eta \circ d
$$

where

$$
\eta \circ d= \begin{cases}\eta:|\eta|_{x_{i}}=0, & \forall i \neq 0, \\ x_{0}^{n+1}\left[d_{i} \sqcup\left(\eta^{\prime} \circ d\right)\right]: \eta=x_{0}^{n} x_{i} \eta^{\prime}, & n \geq 0, i \neq 0 .\end{cases}
$$

(Here $|\eta|_{x_{i}}$ denotes the number of symbols in $\eta$ equivalent to $x_{i}$ and $d_{i}: \xi \mapsto(d, \xi)_{i}$, the ith component of $(d, \xi)$.)

Consequently, if

$$
\eta=x_{0}^{n_{k}} x_{i_{k}} x_{0}^{n_{k-1}} x_{i_{k-1}} \cdots x_{0}^{n_{1}} x_{i_{1}} x_{0}^{n_{0}},
$$

where $i_{j} \neq 0$ for $j=1, \ldots, k$, it follows that

$$
\eta \circ d=x_{0}^{n_{k}+1}\left[d_{i_{k}} \sqcup x_{0}^{n_{k-1}+1}\left[d_{i_{k-1}} \sqcup \cdots x_{0}^{n_{1}+1}\left[d_{i_{1}} \sqcup x_{0}^{n_{0}}\right] \cdots\right]\right] .
$$

Alternatively, for any $\eta \in X^{*}$ one can uniquely define a set of right factors $\left\{\eta_{0}, \eta_{1}, \ldots, \eta_{k}\right\}$ of $\eta$ by the iteration

$$
\eta_{j+1}=x_{0}^{n_{j+1}} x_{i_{j+1}} \eta_{j}, \quad \eta_{0}=x_{0}^{n_{0}}, \quad i_{j+1} \neq 0
$$

so that $\eta=\eta_{k}$ with $k=|\eta|-|\eta|_{x_{0}}$. In which case, $\eta \circ d=\eta_{k} \circ d$, where $\eta_{j+1} \circ d=$ $x_{0}^{n_{j+1}+1}\left[d_{i_{j+1}} \sqcup\left(\eta_{j} \circ d\right)\right]$ and $\eta_{0} \circ d=x_{0}^{n_{0}}$. It was shown in $[15,16]$ that the composition product of two series is always well defined since the family of series $\left\{\eta \circ d: \eta \in X^{*}\right\}$ is locally finite for any fixed $d \in \mathbb{R}^{m}\langle\langle X\rangle\rangle$.

It is easily verified that for any series $c, d, e \in \mathbb{R}^{m}\langle\langle X\rangle\rangle$, the composition product is left distributive over addition. That is,

$$
(c+d) \circ e=c \circ e+d \circ e,
$$

but in general $c \circ(d+e) \neq c \circ d+c \circ e$. An exception is the class of series called linear series. A series $c$ is linear if

$$
\operatorname{supp}(c) \subseteq\left\{\eta \in X^{*}: \eta=x_{0}^{n_{1}} x_{i} x_{0}^{n_{0}}, i \in\{1,2, \ldots, m\}, n_{1}, n_{0} \geq 0\right\}
$$

The composition product is associative, that is, $(c \circ d) \circ e=c \circ(d \circ e)$; hence $\left(\mathbb{R}^{m}\langle\langle X\rangle\rangle, \circ\right)$ forms a semigroup $[4,20]$. In [16], it is shown that the composition product of two locally convergent formal power series is always locally convergent; therefore the set $\mathbb{R}_{L C}^{m}\langle\langle X\rangle\rangle$ is closed under composition, and $\left(\mathbb{R}_{L C}^{m}\langle\langle X\rangle\rangle, \circ\right)$ also forms a semigroup. The central property of the composition product is its relationship to the composition of two Fliess operators. Namely, for any $c \in \mathbb{R}_{L C}^{\ell}\langle\langle X\rangle\rangle$ and $d \in \mathbb{R}_{L C}^{m}\langle\langle X\rangle\rangle$, the cascade connection of two 


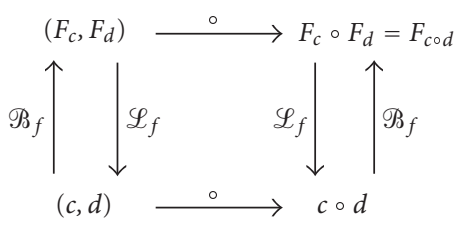

Figure 3.1. The isomorphism between the semigroups $(\mathscr{F}, \circ)$ and $\left(\mathbb{R}_{L C}^{m}\langle\langle X\rangle\rangle, \circ\right)$.

Fliess operators is always another Fliess operator with generating series $c \circ d$, that is,

$$
F_{c} \circ F_{d}=F_{c \circ d}
$$

Therefore, FF forms a semigroup under operator composition when $\ell=m$. The following theorem shows that composition is preserved in a natural sense under the formal LaplaceBorel transform.

Theorem 3.2. Let $X=\left\{x_{0}, x_{1}, \ldots, x_{m}\right\}$. For any $c \in \mathbb{R}_{L C}^{\ell}\langle\langle X\rangle\rangle$ and $d \in \mathbb{R}_{L C}^{m}\langle\langle X\rangle\rangle$,

$$
\begin{gathered}
\mathscr{L}_{f}\left(F_{c} \circ F_{d}\right)=\mathscr{L}_{f}\left(F_{c}\right) \circ \mathscr{L}_{f}\left(F_{d}\right), \\
\mathscr{B}_{f}(c \circ d)=\mathscr{B}_{f}(c) \circ \mathscr{B}_{f}(d) .
\end{gathered}
$$

Proof. The proof follows directly from the definitions. For any well-defined $F_{c}$ and $F_{d}$,

$$
\mathscr{L}_{f}\left(F_{c} \circ F_{d}\right)=\mathscr{L}_{f}\left(F_{c \circ d}\right)=c \circ d=\mathscr{L}_{f}\left(F_{c}\right) \circ \mathscr{L}_{f}\left(F_{d}\right) .
$$

Similarly, for any locally convergent formal power series $c$ and $d$,

$$
\mathscr{B}_{f}(c \circ d)=F_{c \circ d}=F_{c} \circ F_{d}=\mathscr{B}_{f}(c) \circ \mathscr{B}_{f}(d) .
$$

From Theorem 3.2, when $\ell=m$, the formal Laplace-Borel transform provides an isomorphism between the two semigroups $(\mathscr{F}, \circ)$ and $\left(\mathbb{R}_{L C}^{m}\langle\langle X\rangle\rangle\right.$, $)$, as shown in Figure 3.1.

Example 3.3. Let $X=\left\{x_{0}, x_{1}, x_{2}\right\}, F_{c}[u](t)=\cos \left(\int_{0}^{t} u_{1}(t)+u_{2}(t) d t\right)$, and $d=\left(d_{1} d_{2}\right)^{T} \in$ $\mathbb{R}_{L C}^{2}\langle\langle X\rangle\rangle$. Define

$$
F_{e}[u](t)=\left(F_{c} \circ F_{d}\right)[u](t)=\cos \left(\int_{0}^{t} F_{d_{1}}[u](t)+F_{d_{2}}[u](t) d t\right) .
$$

The formal Laplace transform of $F_{e}$ is then

$$
\begin{aligned}
\mathscr{L}_{f}\left[F_{e}\right] & =c \circ d=\sum_{i \geq 0}(-1)^{i}\left(x_{1}+x_{2}\right)^{2 i} \circ d \\
& =\left[-\left(x_{1}+x_{2}\right)^{2}\right]^{\star} \circ d=\frac{1}{1+\left(x_{1}+x_{2}\right)^{2}} \circ d .
\end{aligned}
$$


Example 3.4. Let $X=\left\{x_{0}, x_{1}, \ldots, x_{m}\right\}$ and $c \in \mathbb{R}_{L C}^{m}\langle\langle X\rangle\rangle$. It is easily verified by induction that for $n \geq 1$,

$$
x_{i}^{n} \circ c=\frac{1}{n !}\left(x_{0} c_{i}\right)^{w n}, \quad i=1,2, \ldots, m,
$$

where $(\cdot)^{w n}$ denotes the shuffle power.

Applying the formal Borel transform to both sides of this identity gives

$$
\begin{aligned}
\mathscr{B}_{f}\left[x_{i}^{n} \circ c\right] & =\mathscr{B}_{f}\left[\frac{1}{n !}\left(x_{0} c_{i}\right)^{w n}\right] \\
& =\frac{1}{n !}\left[\mathscr{B}_{f}\left[x_{0} c_{i}\right]\right]^{n} \\
& =\frac{1}{n !}\left[\int_{0}^{t} F_{c_{i}}[u](\tau) d \tau\right]^{n} .
\end{aligned}
$$

Example 3.5. First consider the linear ordinary differential equation

$$
\frac{d^{n} y(t)}{d t^{n}}+\sum_{i=0}^{n-1} a_{i} \frac{d^{i} y(t)}{d t^{i}}=\sum_{i=0}^{n-1} b_{i} \frac{d^{i} u(t)}{d t^{i}}
$$

with initial conditions $y^{(i)}(0)=0, u^{(i)}(0)=0$, and where $a_{i}, b_{i} \in \mathbb{R}$ for $i=0,1, \ldots, n-1$. Integrate both sides of the equation $n$ times and assume there exists a $c \in \mathbb{R}_{L C}\langle\langle X\rangle\rangle$ such that $y=F_{c}[u]$. Applying the formal Laplace transform to both sides of the equation gives

$$
\begin{gathered}
\left(\delta+\sum_{i=0}^{n-1} a_{i} x_{0}^{n-1-i} x_{1}\right) \circ c=\sum_{i=0}^{n-1} b_{i} x_{0}^{n-1-i} x_{1}, \\
\left(1+\sum_{i=0}^{n-1} a_{i} x_{0}^{n-i}\right) c=\sum_{i=0}^{n-1} b_{i} x_{0}^{n-1-i} x_{1} .
\end{gathered}
$$

Therefore,

$$
c=\left(1+\sum_{i=0}^{n-1} a_{i} x_{0}^{n-i}\right)^{-1} \sum_{i=0}^{n-1} b_{i} x_{0}^{n-1-i} x_{1} .
$$

Rephrased in the language of the integral Laplace transform, this is equivalent to

$$
\begin{aligned}
Y(s) & =\left(1+\sum_{i=0}^{n-1} a_{i} \frac{1}{s^{n-i}}\right)^{-1}\left(\sum_{i=0}^{n-1} b_{i} \frac{1}{s^{n-i}}\right) U(s) \\
& =\left(s^{n}+\sum_{i=0}^{n-1} a_{i} s^{i}\right)^{-1}\left(\sum_{i=0}^{n-1} b_{i} s^{i}\right) U(s) .
\end{aligned}
$$

Now consider the nonlinear differential equation

$$
\frac{d^{n} y(t)}{d t^{n}}+\sum_{i=0}^{n-1} a_{i} \frac{d^{i} y(t)}{d t^{i}}+\sum_{j=2}^{k} p_{j} u(t) y^{j}(t)=\sum_{i=0}^{n-1} b_{i} \frac{d^{i} u(t)}{d t^{i}}
$$


with $y^{(i)}(0)=0, u^{(i)}(0)=0$, and where $a_{i}, b_{i}, p_{j} \in \mathbb{R}$ for $i=0,1, \ldots, n-1$ and $j=2, \ldots, k$. Again integrate both sides of the equation $n$ times and assume $y=F_{c}[u]$. Applying the formal Laplace transform gives

$$
\left(1+\sum_{i=0}^{n-1} a_{i} x_{0}^{n-i}\right) c+\sum_{j=2}^{k} p_{j} x_{0}^{n-1} x_{1}\left(c^{\uplus j}\right)=\sum_{i=0}^{n-1} b_{i} x_{0}^{n-1-i} x_{1}
$$

As in [12], a recursive procedure can be applied to solve the algebraic equation iteratively so that

$$
c=c_{1}+c_{2}+\cdots
$$

with

$$
c_{1}=\left(1+\sum_{i=0}^{n-1} a_{i} x_{0}^{n-i}\right)^{-1} \sum_{i=0}^{n-1} b_{i} x_{0}^{n-1-i} x_{1}
$$

and for $n \geq 2$

$$
c_{n}=\left(1+\sum_{i=0}^{n-1} a_{i} x_{0}^{n-i}\right)^{-1} x_{0}^{n-1} x_{1} \sum_{j=2}^{k} p_{j} \sum_{\substack{\nu_{1} \geq 1, \ldots, \nu_{j} \geq 1 \\ \nu_{1}+v_{2}+\cdots+v_{j}=n}} c_{\nu_{1}} \sqcup c_{\nu_{2}} \sqcup \cdots \sqcup c_{\nu_{j}} .
$$

\section{Operational calculus for the output response of Fliess operators}

In $[7,12,19]$, an operational calculus was developed to compute the output response of an analytic nonlinear system represented by a Fliess operator. In this section, the formal Laplace-Borel transform pair in conjunction with the composition product are employed to produce a clear and compact interpretation of this methodology.

Theorem 4.1. Let $F_{c} \in \mathscr{F}$ and let $u$ be an analytic function with formal Laplace transform $c_{u} \in \mathbb{R}_{L C}^{m}\left\langle\left\langle X_{0}\right\rangle\right\rangle$. Then the output function $y=F_{c}[u]$ is analytic and has formal Laplace transform $c_{y}=c \circ c_{u} \in \mathbb{R}_{L C}^{\ell}\left\langle\left\langle X_{0}\right\rangle\right\rangle$.

Proof. The analyticity of $y$ follows from [24, Lemma 2.3.8]. Let $c_{y}$ denote its formal Laplace transform. Local convergence is preserved under the composition product; therefore the formal power series $c \circ c_{u}$ is locally convergent, and, for any admissible input $v \in B_{p}^{m}(R)\left[t_{0}, t_{0}+T\right]$

$$
y=F_{c_{y}}[v]=F_{c}\left[F_{c_{u}}[v]\right]=F_{c^{\circ} c_{u}}[v] .
$$

Applying the formal Laplace transform to both sides of the equation gives $c_{y}=c \circ c_{u}$.

The following examples further illustrate the applications of this result for computing the output response of an analytic input-output system.

Example 4.2. Consider the linear time-invariant system $y(t)=\int_{0}^{t} h(t-\tau) u(\tau) d \tau$, where $h$ is analytic at $t=0$. Then $y=F_{c}[u]$ with $\left(c, x_{0}^{k} x_{1}\right)=h^{(k)}(0), k \geq 0$, and zero otherwise. 
12 The formal Laplace-Borel transform of Fliess operators

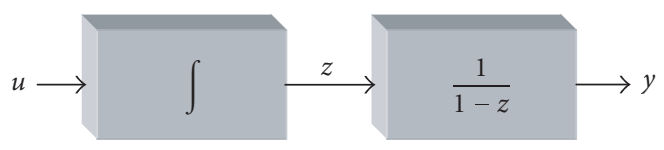

Figure 4.1. A simple Wiener system.

If $u(t)=\sum_{k \geq 0}\left(c_{u}, x_{0}^{k}\right) t^{k} / k$ !, then it follows that $y(t)=\sum_{n \geq 0}\left(c_{y}, x_{0}^{n}\right) t^{n} / n !$, where

$$
\begin{aligned}
c_{y} & =c \circ c_{u} \\
& =\sum_{k \geq 0}\left(c, x_{0}^{k} x_{1}\right) x_{0}^{k} x_{1} \circ c_{u} \\
& =\sum_{k \geq 0}\left(c, x_{0}^{k} x_{1}\right) x_{0}^{k+1} c_{u} .
\end{aligned}
$$

Therefore,

$$
\left(c_{y}, x_{0}^{n}\right)=\sum_{k=0}^{n-1}\left(c, x_{0}^{k} x_{1}\right)\left(c_{u}, x_{0}^{n-1-k}\right), \quad n \geq 1,
$$

which is the conventional convolution sum.

Example 4.3. Consider the simple Wiener system shown in Figure 4.1 where $z(0)=0$. The mapping $u \mapsto y$ can be written as

$$
y(t)=\sum_{n=0}^{\infty}\left(E_{x_{1}}[u](t)\right)^{n}=\sum_{n=0}^{\infty} F_{x_{1} \amalg n}[u](t)=\sum_{n=0}^{\infty} n ! E_{x_{1}^{n}}[u](t) .
$$

Therefore $y=F_{c}[u]$ with $c=\sum_{n \geq 0} n ! x_{1}^{n}$. When $u(t)=t^{k} / k !$, for example, the formal Laplace transform of $u$ is $c_{u}=x_{0}^{k}$. From Theorem 4.1 and (3.14) it follows that

$$
c_{y}=\sum_{n=0}^{\infty} n ! x_{1}^{n} \circ x_{0}^{k}=\sum_{n=0}^{\infty}\left(x_{0}^{k+1}\right)^{\varpi n}=\sum_{n=0}^{\infty} \frac{((k+1) n) !}{((k+1) !)^{n}} x_{0}^{(k+1) n} .
$$

Consequently, the output response is

$$
y(t)=\sum_{n=0}^{\infty} \frac{((k+1) n) !}{((k+1) !)^{n}} \frac{t^{(k+1) n}}{((k+1) n) !}=\sum_{n=0}^{\infty} \frac{t^{(k+1) n}}{((k+1) !)^{n}}=\frac{1}{1-t^{k+1} /(k+1) !} .
$$

\section{Conclusion}

In this paper, the formal Laplace-Borel transform of a Fliess operator is defined and developed. This concept provides an isomorphism between the semigroup of all Fliess operators under composition and the semigroup of all locally convergent formal power series under the composition product. As an application in system theory, an explicit relationship is derived between the formal Laplace-Borel transforms of the input and output functions of a Fliess operator, which is a compact interpretation of the operational calculus of Fliess. 


\section{References}

[1] J. Berstel and C. Reutenauer, Les Séries Rationelles et Leurs Langages, Springer, Paris, 1984.

[2] A. Cheng and K. Morris, Well-posedness of boundary control systems, SIAM Journal on Control and Optimization 42 (2003), no. 4, 1244-1265.

[3] E. Delaleau and J. Rudolph, An intrinsic characterization of properness for linear time-varying systems, Journal of Mathematical Systems, Estimation, and Control 5 (1995), no. 1, 1-18.

[4] A. Ferfera, Combinatoire du Monoïde Libre Appliquée à la Composition et aux Variations de Certaines Fonctionnelles Issues de la Théorie des Systèmes, Ph.D. thesis, Thèse de 3ème Cycle, Université de Bordeaux I, Bordeaux, 1979.

[5] __ Combinatoire du monoïde libre et composition de certains systèmes non linéaires, Systems Analysis (Conf., Bordeaux, 1978), Astérisque, vol. 75-76, Société Mathématique de France, Paris, 1980, pp. 87-93.

[6] M. Fliess, Un outil algebraique: les séries formaelles non commutatives, Mathematical Systems Theory (Proceedings of International Symposium, Udine, June, 1975), Lecture Notes in Economics and Mathematical Systems, vol. 131, Springer, Berlin, 1976, pp. 122-148.

[7] __ Fonctionnelles causales non linéaires et indéterminées non commutatives, Bulletin de la Société Mathématique de France 109 (1981), no. 1, 3-40.

[8] _ Réalisation locale des systèmes non linéaires, algèbres de Lie filtrées transitives et séries génératrices non commutatives, Inventiones Mathematicae 71 (1983), no. 3, 521-537.

[9] _ Une interprétation algébrique de la transformation de Laplace et des matrices de transfert, Linear Algebra and Its Applications 203-204 (1994), 429-442.

[10] __ Some properties of linear recurrent error-control codes: a module-theoretic approach, Proceedings of 15th International Symposium on Mathematical Theory of Networks and Systems, Indiana, August 2002.

[11] M. Fliess, C. Join, and H. Sira-Ramírez, Robust residual generation for linear fault diagnosis: an algebraic setting with examples, International Journal of Control 77 (2004), no. 14, 1223-1242.

[12] M. Fliess, M. Lamnabhi, and F. Lamnabhi-Lagarrigue, An algebraic approach to nonlinear functional expansions, IEEE Transactions on Circuits and Systems 30 (1983), no. 8, 554-570.

[13] W. S. Gray and Y. Li, Fliess operators in cascade and feedback systems, Proceedings of the 36th Conference on Information Sciences and Systems, New Jersey, March 2002, pp. 173-178.

[14]__ Generating series for nonlinear cascade and feedback systems, Proceedings of the 41st IEEE Conference on Decision and Control, Nevada, December 2002, pp. 2720-2725.

[15] _ Interconnected systems of Fliess operators, Proceedings of the 15th International Symposium on Mathematical Theory of Networks and Systems, Indiana, August 2002.

[16] _ Generating series for interconnected analytic nonlinear systems, SIAM Journal on Control and Optimization 44 (2005), no. 2, 646-672.

[17] W. S. Gray and Y. Wang, Fliess operators on $L_{p}$ spaces: convergence and continuity, Systems \& Control Letters 46 (2002), no. 2, 67-74.

[18] M. L. J. Hautus, The formal Laplace transform for smooth linear systems, Mathematical Systems Theory (Proceedings of International Symposium, Udine, June, 1975), Lecture Notes in Economics and Mathematical Systems, vol. 131, Springer, Berlin, 1976, pp. 29-47.

[19] M. Lamnabhi, A new symbolic calculus for the response of nonlinear systems, Systems \& Control Letters 2 (1982), no. 3, 154-162.

[20] Y. Li, Generating series of interconnected nonlinear systems and the formal Laplace-Borel transform, Ph.D. thesis, Old Dominion University, Virginia, 2004.

[21] M. Lothaire, Combinatorics on Words, Cambridge University Press, Cambridge, 1983.

[22] V. H. N. Minh, Evaluation transform, Theoretical Computer Science 79 (1991), no. 1, part A, 163-177.

[23] B. Y. Sternin and V. E. Shatalov, Borel-Laplace Transform and Asymptotic Theory, CRC Press, Florida, 1996. 
14 The formal Laplace-Borel transform of Fliess operators

[24] Y. Wang, Algebraic differential equations and nonlinear control systems, Ph.D. thesis, Department of Mathematics, Rutgers University, New Jersey, 1990.

Yaqin Li: Department of Electrical and Computer Engineering, University of Memphis, Memphis, TN 38152, USA

E-mail address: yaqinli@memphis.edu

W. Steven Gray: Department of Electrical and Computer Engineering, Old Dominion University, Norfolk, VA 23529, USA

E-mail address: gray@ece.odu.edu 


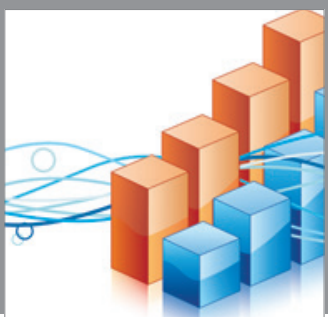

Advances in

Operations Research

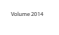

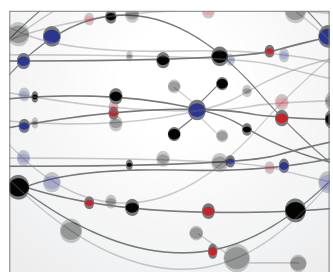

\section{The Scientific} World Journal
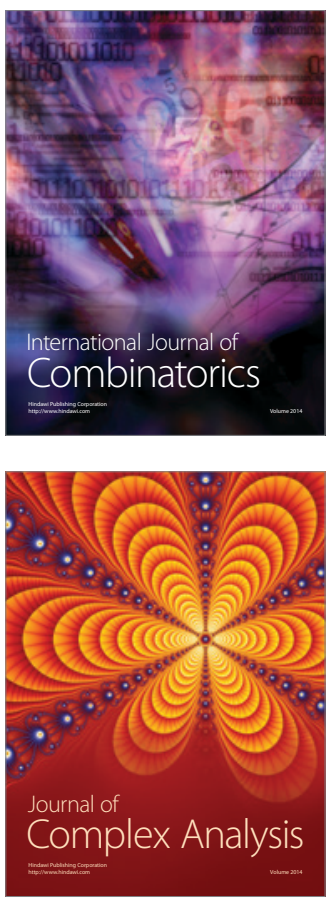

International Journal of

Mathematics and

Mathematical

Sciences
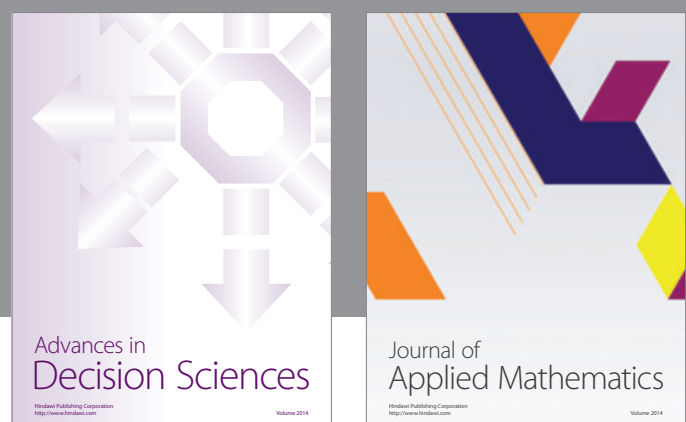

Journal of

Applied Mathematics
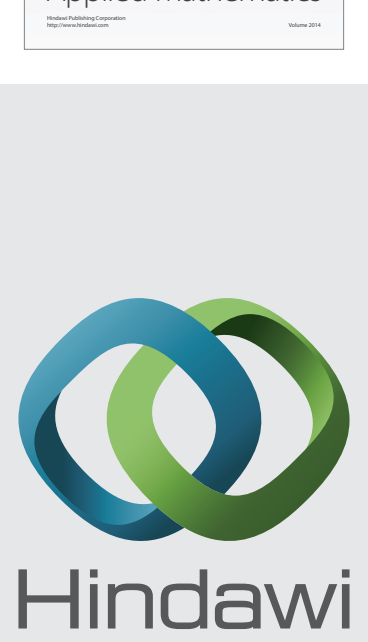

Submit your manuscripts at http://www.hindawi.com
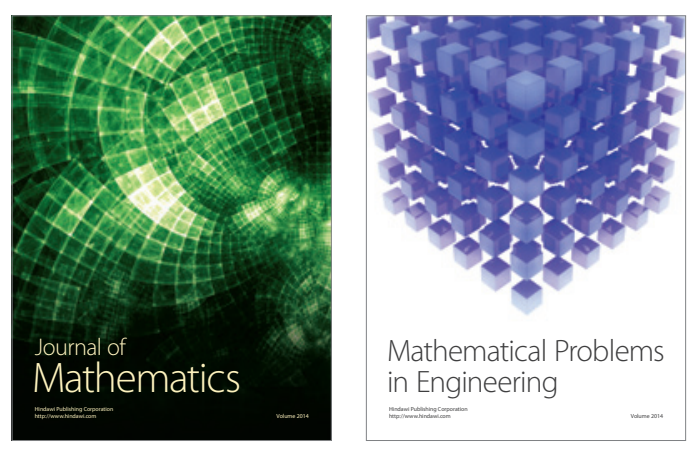

Mathematical Problems in Engineering
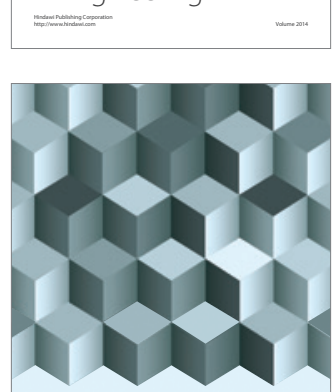

Journal of

Function Spaces
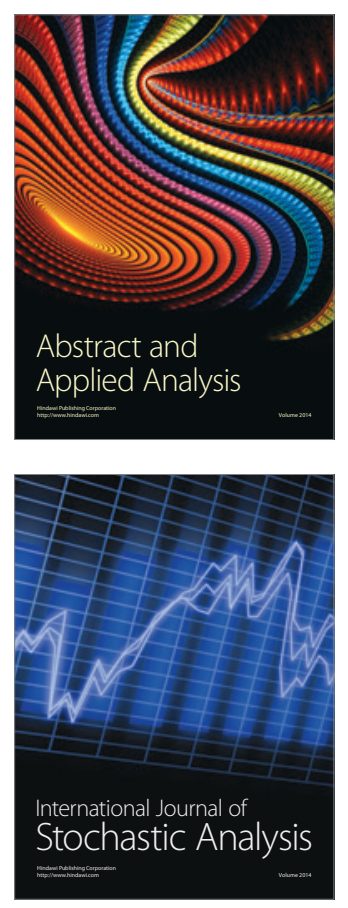

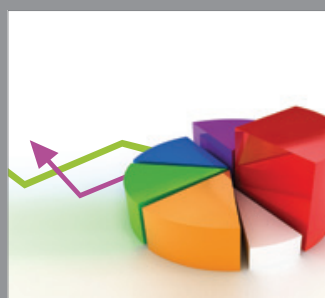

ournal of

Probability and Statistics

Promensencen
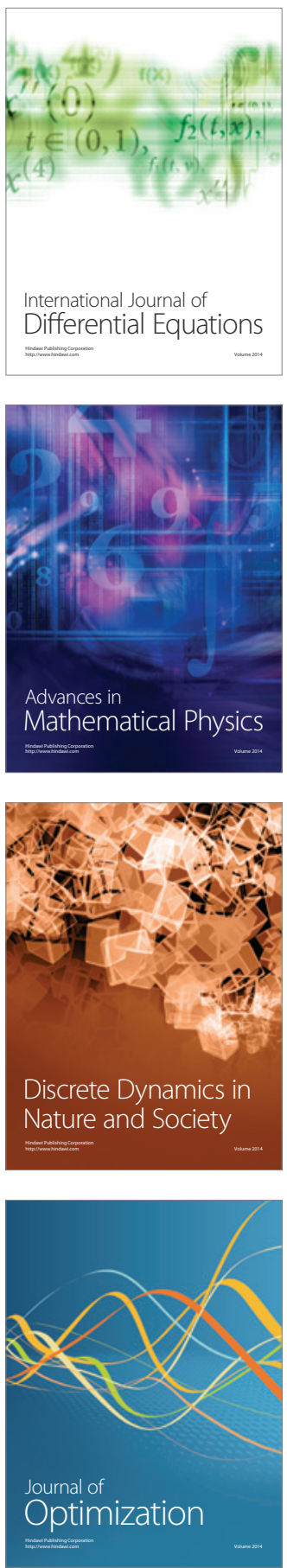